\title{
Erythropoietin and sildenafil protect against ischemia/reperfusion injury following testicular torsion in adult rats
}

\author{
IOANNIS D. KOSTAKIS ${ }^{1,2}$, NICK ZAVRAS ${ }^{3}$, CHRISTOS DAMASKOS $^{1,2}$, STRATIGOULA SAKELLARIOU ${ }^{4}$, \\ PENELOPE KORKOLOPOULOU ${ }^{4}$, EVANGELOS P. MISIAKOS ${ }^{3}$, PETROS TSAPARAS $^{1,2}$, \\ GEORGE VAOS $^{5}$ and THEODOROS KARATZAS ${ }^{1,2}$
}

\begin{abstract}
${ }^{1}$ Laboratory of Experimental Surgery and Surgical Research 'N.S. Christeas', National and Kapodistrian University of Athens, Medical School; ${ }^{2}$ Second Department of Propedeutic Surgery, 'Laiko' General Hospital, National and Kapodistrian University of Athens, Medical School, 11527 Athens; ${ }^{3}$ Third Department of Surgery, Attikon University Hospital, National and Kapodistrian University of Athens, Medical School, 12462 Athens; ${ }^{4}$ First Department of Pathology, National and Kapodistrian University of Athens, Medical School, 11527 Athens; ${ }^{5}$ Department of Pediatric Surgery, Alexandroupolis University Hospital, Democritus University of Thrace, Medical School, 68100 Alexandroupolis, Greece
\end{abstract}

Received March 30, 2016; Accepted September 22, 2016

DOI: $10.3892 / \mathrm{etm} .2017 .4441$

\begin{abstract}
Testicular torsion/detorsion causes severe tissue damage due to ischemia/reperfusion injury. The present study investigated the protective effect of erythropoietin and sildenafil against ischemia/reperfusion injury following unilateral testicular torsion/detorsion in adult rats. A total of 28 adult male rats were included, and were divided into the following groups: Group A ( $n=5)$, sham operated; groups B ( $n=5), C(n=5), D(n=5)$ and $\mathrm{E}(\mathrm{n}=8)$, undergoing right testis torsion and detorsion after 90 min. Group B received no drug treatment. Rats in the groups $\mathrm{C}$ and D received low-dose $(1,000 \mathrm{IU} / \mathrm{kg})$ or high-dose $(3,000 \mathrm{IU} / \mathrm{kg})$ erythropoietin, while those in group $\mathrm{E}$ received sildenafil $(0.7 \mathrm{mg} / \mathrm{kg})$, through intraperitoneal injection after $60 \mathrm{~min}$ of torsion. The right testis was extracted $24 \mathrm{~h}$ after detorsion, and the tissue was subjected to histopathological examination and immunohistochemical assessment of cleaved caspase-3 expression. Histological alterations and the quality of spermatogenesis were scored according to the Cosentino and the Johnsen scoring systems, respectively. The results demonstrated normal testicular architecture in group A, while the other groups showed ischemic cellular damages, with the worst scores observed in group B. Groups D and E presented better scores compared with group C. Regarding the quality of spermatogenesis, the best scores were observed in group $\mathrm{A}$, and the worst in group B. Groups C, D and E presented similar results, which were improved in comparison to group B, however, not
\end{abstract}

Correspondence to: Dr Ioannis D. Kostakis, Laboratory of Experimental Surgery and Surgical Research 'N.S. Christeas', National and Kapodistrian University of Athens, Medical School, 15b Agiou Thoma Street, 11527 Athens, Greece

E-mail: i.d.kostakis@gmail.com

Key words: erythropoietin, sildenafil, ischemia/reperfusion injury, testicular torsion, testis, rats compared to group A. Furthermore, cleaved caspase-3 levels were lower in groups A, D and E, with equal results observed. Group $C$ had higher levels of cleaved caspase-3 compared with these groups, but lower than group B, which presented the highest cleaved caspase-3 levels. In conclusion, erythropoietin and sildenafil protect testis from ischemia/reperfusion injury by decreasing cellular damage and attenuating apoptosis.

\section{Introduction}

Testicular torsion is caused by the rotation of the spermatic cord, initially resulting in the interruption of testicular venous flow and then of arterial flow, which consequently leads to ischemia of the testis (1-9). It is an emergent condition that mainly affects the pediatric and young adult male population and requires urgent surgical detorsion, since it may result in loss of the affected testicle if not treated promptly by surgery (1-9). Significant ischemic damage of the testis occurs after 4-8 $\mathrm{h}$ of torsion $(2,3,6)$, and the salvage rates reduces as the interval between torsion and detorsion increases (1-9), with rates of $90 \%$ after $6 \mathrm{~h}, 50 \%$ after $12 \mathrm{~h}$ and $10 \%$ after $24 \mathrm{~h}(1,3,7)$. Germ cell death is induced by testicular ischemia, and is mainly attributed to the reduction in oxygen supply relative to metabolic demands, accumulation of toxic metabolites and depletion of the stored cellular energy. The restoration of perfusion of the testis subsequent to detorsion leads to increased production of reactive oxygen species, which damage the DNA, proteins and lipids of testicular cells $(1,4,5,8,9)$. Reperfusion also results in the production of various proinflammatory cytokines, including interleukin- $1 \beta$ and tumor necrosis factor- $\alpha$, which cause recruitment of macrophages and neutrophils that infiltrate the testicular parenchyma $(4,5,8,9)$. These pathophysiological changes cause apoptosis of germinal cells $(4,8,9)$ and impair the function of Sertoli cells (9), leading to deterioration of spermatogenesis and testicular atrophy $(1,4,5,9)$.

Erythropoietin and sildenafil are considered to protect against ischemia/reperfusion injury in various tissues and 
organs $(10,11)$. Erythropoietin is a glycoprotein that regulates red cell production, and functions as a rescue factor of red cells by increasing their survival and inhibiting their apoptosis in vitro (12). Sildenafil is an inhibitor of phosphodiesterase-5 and is widely used as a new class vasoactive drug that has a powerful effect against ischemia/reperfusion injury in several tissues of different animal models $(13,14)$. In a previous study by our group (15), the protective effect of intraperitoneal injection of erythropoietin and sildenafil in adult rats that underwent testicular torsion and detorsion was investigated and compared. The study demonstrated that both erythropoietin and sildenafil treatment resulted in milder histological changes as compared with the control, with sildenafil having a possibly enhanced action (15). However, only low-dose erythropoietin treatment was previously investigated.

Thus, the present study aimed to investigated the protective role of low- and high-dose erythropoietin, as well as of sildenafil, against ischemia/reperfusion injury after testicular torsion and detorsion in rats. In addition, the effects of erythropoietin and sildenafil on spermatogenesis and cell apoptosis in the rat testis were examined, which had not been investigated in our previous study.

\section{Materials and methods}

Animals. In the present study, 28 adult male Wistar Albino rats between 12 and 14 weeks of age were used. Their weight ranged between 250 and $300 \mathrm{~g}$. The animals were provided by the Hellenic Pasteur Institute (Athens, Greece), and were acclimatized in our laboratory for 1 week before the onset of the experiment. The housing conditions were standardized to provide 12-h light and dark cycles, temperature of $22 \pm 2^{\circ} \mathrm{C}$ and relative humidity of $60 \%$. All animals received analgesia with carprofen $(0.08 \mathrm{ml} / \mathrm{kg}$; Rimadyl; Zoetis Inc., Florham Park, NJ, USA) and chemoprophylaxis with enrofloxacin (10 mg/kg; Baytril; Bayer AG, Leverkusen, Germany) subcutaneously preoperatively. An intramuscular injection of ketamine (100 mg/kg; Institute of Pharmaceutical Research and Technology, Attica, Greece) and xylazine (5 mg/kg; Rompun; Bayer AG) was used for anesthesia in each animal. All rats breathed spontaneously during the surgical procedures. The skin of their scrotum was shaved and scrubbed with Betadine (Lavipharm SA, Attica, Greece) solution. All procedures were performed under sterile conditions. The experimental protocols conformed to the ethical recommendations of the European Union Directive 2010/63/EU for animal experiments, and were approved by the Veterinary Directorate of the Region of Attica and the Ethics Committee of the Medical School of the University of Athens (Athens, Greece).

Torsion/detorsion animal model. A rat model of testicular torsion and detorsion was established in the present study. Briefly, a right vertical paramedian incision was made on the scrotum and the right testis was exposed. The testis was rotated $720^{\circ}$ clockwise to perform torsion, then fixed on the internal spermatic fascia using 4/0 polyglycolic acid suture and placed back in its anatomical position. The scrotal incision was closed with $4 / 0$ silk suture. After $90 \mathrm{~min}$, the scrotum was reopened and the right testis was exposed, derotated to its normal position and placed back in its anatomical position. The scrotum was closed again with $4 / 0$ silk suture. All animals were sacrificed after $24 \mathrm{~h}$ from detorsion. The scrotum was reopened to extract the right testis, and the tissue was subjected to for histopathological and immunohistochemical analyses.

Animal groups. The animals were randomly divided into five groups. Group A included 5 sham-operated rats in which no torsion was performed. All the animals in the other four groups underwent all the stages of the aforementioned surgical procedure to establish a torsion/detorsion model. Group B included 5 rats, which received no drug treatment. Animals in groups $\mathrm{C}, \mathrm{D}$ and $\mathrm{E}$ received an intraperitoneal drug injection after 60 minutes of torsion. Groups C and D included 5 rats each, and were administered a low dose $(1,000 \mathrm{IU} / \mathrm{kg})$ or high dose $(3,000 \mathrm{IU} / \mathrm{kg})$ of recombinant human erythropoietin was administered. Group E included 8 rats that were administered sildenafil $(0.7 \mathrm{mg} / \mathrm{kg})$.

Drugs and doses. Recombinant human erythropoietin was used in the form of injections (Aranesp, Amgen, Holland). A dose of $1,000 \mathrm{IU} / \mathrm{kg}$ was injected intraperitoneally in the rats of group $\mathrm{C}$ (low-dose erythropoietin group) and a dose of 3,000 IU/kg was injected intraperitoneally in the rats of group D (high-dose erythropoietin group). Sildenafil in the form of commercially packed tablets (Viagra; Pfizer, Walton Oaks, UK) was also obtained. Each tablet contained $100 \mathrm{mg}$ of sildenafil. The tablets were grinded and dissolved in sterile $0.9 \% \mathrm{w} / \mathrm{v} \mathrm{NaCl}$ solution, forming a $20 \% \mathrm{w} / \mathrm{v}$ sildenafil solution. A dose of $0.7 \mathrm{mg} / \mathrm{kg}$ was injected intraperitoneally in the rats of group $\mathrm{E}$ (sildenafil group). These doses of erythropoietin and sildenafil have been reported by previous studies (16-22).

Histopathological analysis. The right testis of each animal was placed into $10 \%$ formalin solution immediately following its extraction, and tissue specimens were obtained from the testis of each animal. The specimens were then embedded in paraffin and cut into sections with thickness of $5 \mu \mathrm{m}$. Subsequently, hematoxylin and eosin staining of the sections was conducted, followed by examination with a light microscope. As shown in Table I, the grading system proposed by Cosentino et al (23) was used to evaluate any histological changes in the testes that were caused by ischemia and reperfusion. Ischemia and reperfusion resulted in tissue damage with varying degrees of severity observed in different areas of the rat testis. Therefore, a different score was assigned to each testicular area, and the final Cosentino's grade for each testis was calculated by multiplying the grade of each area by the percentage of the total surface that it occupied. The presence of pyknotic nuclei surrounded by apoptotic bodies (debris) was defined as apoptosis, whereas the presence of disrupted cell membranes was defined as necrosis.

Spermatogenesis in the tissue specimens of the testes was also evaluated according to the scoring system proposed by Johnsen (24), as described in Table II. Ischemia and reperfusion resulted in alterations in spermatogenesis with different severity observed in different areas of each testis. Therefore, a 
Table I. Histological grading system of testicular damage observed in tissue samples, as proposed by Cosentino et al (23).

\begin{tabular}{ll} 
Grade & Characteristics \\
\hline I & $\begin{array}{l}\text { Normal testicular architecture with an orderly } \\
\text { arrangement of germinal cells }\end{array}$ \\
II & $\begin{array}{l}\text { Injury showed less orderly, non-cohesive germinal } \\
\text { cells and closely packed seminiferous tubules }\end{array}$ \\
III $\quad \begin{array}{l}\text { Injury exhibited disordered sloughed germinal } \\
\text { cells, with reduced size of pyknotic nuclei and less } \\
\text { distinct seminiferous tubule borders }\end{array}$ \\
IV $\quad \begin{array}{l}\text { Injury exhibited seminiferous tubules that were } \\
\text { closely packed with coagulative necrosis of the } \\
\text { germinal cells }\end{array}$
\end{tabular}

different score was assigned to each testicular different area, and the final Johnsen's score for each testis was determined by multiplying the grade of each area by the percentage of the total surface that it occupied. Histological changes and spermatogenesis scoring were assessed in random order by a pathologist blinded to the study groups.

Immunohistochemical analysis. Caspase-3 is one of the proteins activated during cell apoptosis and necrosis (25-28). The present study used this protein as a marker of cell death by assessing the immunoreactivity of cleaved caspase-3. The percentage of positive cells was evaluated in five random areas from each section and the mean value was calculated for each animal. Immunohistochemistry was performed on paraffin-embedded $5-\mu \mathrm{m}$ sections using the cleaved caspase-3 (Asp175; 5A1E) rabbit monoclonal antibody (1:300 dilution; Cell Signaling Technology, Inc., Danvers, MA, USA), using a two-step peroxidase conjugated polymer technique (Dako Envision kit (Dako, Carpinteria, CA, USA). Briefly, the tissue sections were deparaffinized, rehydrated and treated with a hydrogen peroxide solution for $20 \mathrm{~min}$. Antigen retrieval was performed by heating the slides in a microwave oven in Tris-EDTA buffer (pH 9) for a total of $10 \mathrm{~min}$. Subsequently, the sections were incubated overnight at $4^{\circ} \mathrm{C}$ with the caspase-3 antibody. Chromogenic detection was achieved by a diaminobenzidine substrate (BioGenex, San Ramon, CA, USA), which was applied for $5 \mathrm{~min}$, followed by counterstaining with Harris hematoxylin. Finally, the sections were dehydrated and coverslipped. Cleaved caspase-3 immunoreactivity was assessed in random order by a pathologist blinded to the study groups.

Statistical analysis. Shapiro-Wilk test was used for the assessment of the normality of data distribution, while Levene's test was used to assess the homogeneity of variance. Analysis of variance with the Bonferroni correction was performed for comparisons among groups. Spearman's rank correlation coefficient was performed for the evaluation of correlation between quantitative parameters. SPSS version 22.0 (IBM SPSS, Amronk, NY, USA) was used for data analysis. The results were considered to be statistically significant when the P-value was $<0.05$.
Table II. Spermatogenesis scoring system observed in the testicular tissue of rats, as proposed by Johnsen (24).

\begin{tabular}{|c|c|}
\hline Score & Characteristics \\
\hline 10 & $\begin{array}{l}\text { Complete spermatogenesis and normally organized } \\
\text { tubules }\end{array}$ \\
\hline 9 & $\begin{array}{l}\text { Numerous spermatozoa present, but the germinal } \\
\text { epithelium is disorganized }\end{array}$ \\
\hline 8 & Only a few spermatozoa present in the section \\
\hline 7 & No spermatozoa, but numerous spermatids present \\
\hline 6 & Only a few spermatids present \\
\hline 5 & $\begin{array}{l}\text { No spermatozoa or spermatids, but numerous } \\
\text { spermatocytes present }\end{array}$ \\
\hline 4 & Only a few spermatocytes present \\
\hline 3 & Only spermatogonia present \\
\hline 2 & No germ cells, but only Sertoli cells present \\
\hline 1 & No germ cells and no Sertoli cells present \\
\hline
\end{tabular}

\section{Results}

Histological changes (Cosentino's score). Histological changes in the testicular tissue of rats in the study groups were assessed by Cosentino's score. Indicative histological images from each group are shown in Fig. 1. Histological examination of testes in group A (sham-operated rats) demonstrated normal testicular architecture with orderly arranged germinal cells. These features corresponded to grade I according to Cosentino's scoring system, and all rats in group A had a total score of 1. By contrast, rats in group B (group subjected to torsion/detorsion of testis without pharmaceutical intervention; mean grade, 3.86; range, 3.6-4) presented severe histological lesions in their testicular tissue. Specifically, the histological examination of testes revealed closely packed seminiferous tubules and coagulative necrosis of the germinal cells in the largest part of the sample, along with smaller areas characterized by apoptotic germinal cells and ill-defined borders of seminiferous tubules. Rats in group C (torsion/detorsion of testis with administration of low-dose erythropoietin; mean grade, 3.22; range, 2.95-3.45) revealed moderate histological lesions in the greatest part of the testes. The tubule borders of seminiferous tubules were ill-defined and the germinal cells appeared to be disordered and sloughed with reduced size of pyknotic nuclei. However, there were large areas with more severe lesions characterized by closely packed seminiferous tubules and necrotic germinal cells, as well as small areas with milder lesions characterized by closely packed, but better defined, seminiferous tubules and non-cohesive germinal cells. Rats in group D (torsion/detorsion of testis with administration of high-dose erythropoietin; mean grade, 2.56; range, 2.4-2.75) and group $\mathrm{E}$ (torsion/detorsion of testis with administration of sildenafil; mean grade, 2.67; range, 2.35-2.85) revealed milder histological lesions in comparison with other groups. The greatest part of the testes revealed ill-defined seminiferous tubules and disordered sloughed germinal cells with reduced size of pyknotic nuclei. There were also large areas with 
$\mathbf{A}$

B

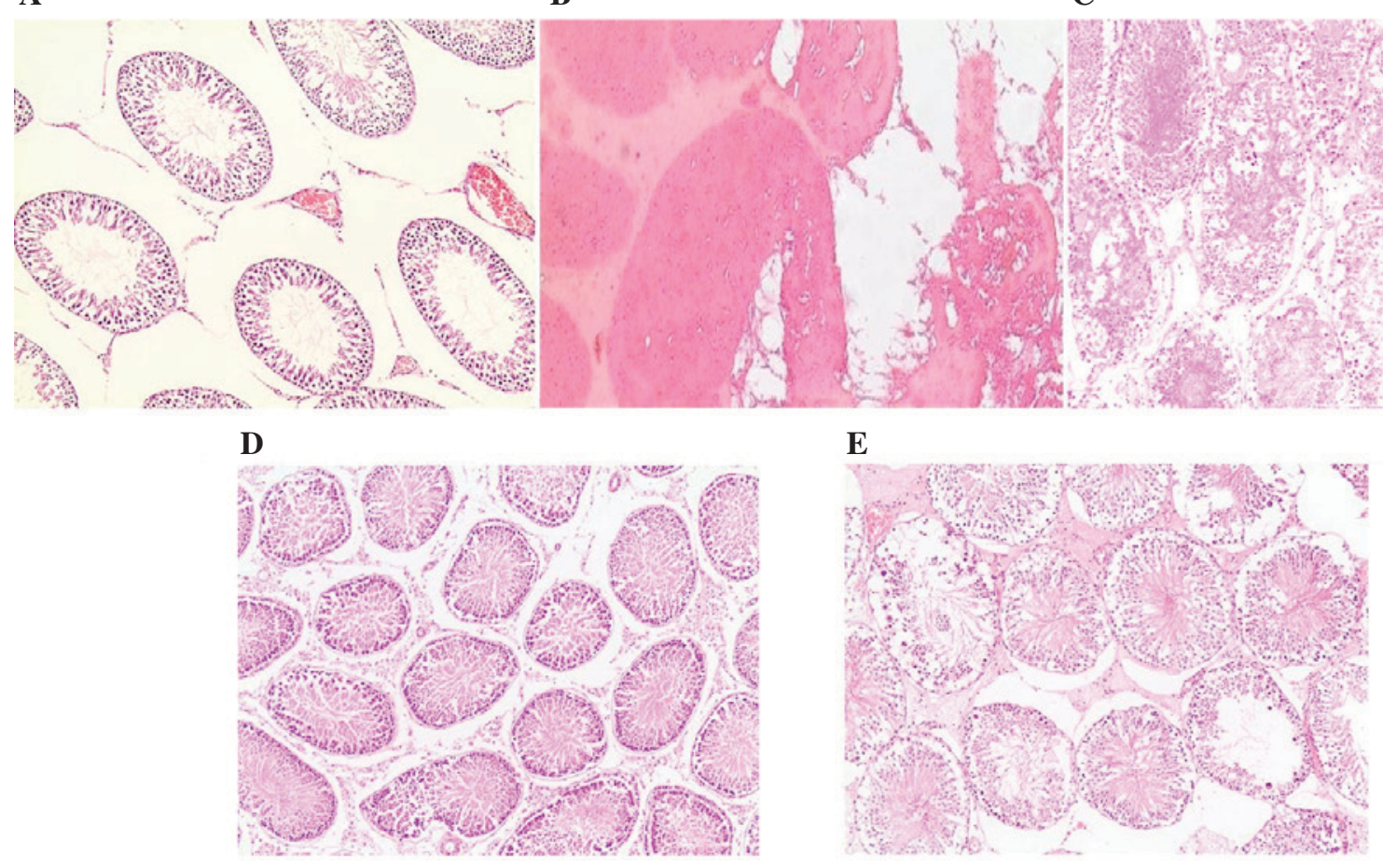

Figure 1. Histological images of hematoxylin and eosin staining of rat testicular tissues in the (A) sham-operated, (B) torsion/detorsion without treatment, (C) torsion/detorsion + low-dose erythropoietin,(D) torsion/detorsion + high-dose erythropoietin, and (E) torsion/detorsion + sildenafil groups (original magnification, x100).

less severe histological changes, having closely packed, but better defined, seminiferous tubules and disorderly arranged germinal cells.

Upon comparison of the grades observed in the five groups, statistically significant differences were identified among the groups. More specifically, Group A demonstrated significantly lower grade results than all the other groups $(\mathrm{P}<0.0001)$. By contrast, group B showed significantly higher grade results compared with all other groups $(\mathrm{P} \leq 0.0001)$. In addition, group $\mathrm{C}$ rats exhibited lower grades in comparison with group $\mathrm{B}(\mathrm{P}=0.0001)$, but higher grades compared with the rest of the groups $(\mathrm{P}<0.0001)$. However, the Cosentino's scores of groups $\mathrm{D}$ and $\mathrm{E}$ were not significantly different $(\mathrm{P}>0.99)$. Furthermore, group D and E rats presented lower grades compared with groups $\mathrm{B}$ and $\mathrm{C}$, whereas higher grades were observed compared with group A (all $\mathrm{P} \leq 0.0001$ ). Lower grades indicated milder histological damage and higher grades indicated more severe histological damage.

Spermatogenesis (Johnsen's score). Spermatogenesis was assessed histopathologically by Johnsen's score. Rats in group A (sham-operated) exhibited normal or slightly affected spermatogenesis (mean score, 9.8). Specifically, 4 rats showed complete spermatogenesis and normally organized seminiferous tubules (Johnsen's score 10), while 1 rat showed presence of numerous spermatozoa, but with disorganized germinal epithelium (Johnsen's score 9). By contrast, rats in group B (torsion/detorsion of testis without pharmaceutical treatment) demonstrated severely damaged spermatogenesis (mean score, 3). Specifically, 3 rats had only spermatogonia present (Johnsen's score 3), 1 rat had only a few spermatocytes present
(Johnsen's score 4) and 1 rat had no germ cells, but only Sertoli cells were present (Johnsen's score 2). Rats in groups C, D and E that were treated with erythropoietin or sildenafil showed only mildly damaged spermatogenesis. In group C (torsion/detorsion of testis with administration of low-dose erythropoietin), 3 rats had only a few spermatozoa present (Johnsen's score 8), 1 rat presented numerous spermatozoa with disorganized germinal epithelium (Johnsen's score 9), and 1 rat had no spermatozoa, but numerous spermatids were present (Johnsen's score 7; mean score, 8). In group D (torsion/detorsion of testis with administration of high-dose erythropoietin), 3 rats showed the presence of numerous spermatozoa with disorganized germinal epithelium (Johnsen's score 9), and 2 rats had only a few spermatozoa present (Johnsen's score 8; mean score, 8.6). Finally, in group E (torsion/detorsion of testis with administration of sildenafil), 4 rats showed the presence of numerous spermatozoa with disorganized germinal epithelium (Johnsen's score 9), while 4 rats had only a few spermatozoa present (Johnsen's score 8; mean score, 8.5).

Upon comparison of the scores among the five groups, certain statistically significant differences were identified. Group A exhibited higher Johnsen's scores in comparison with any other group (A vs. $\mathrm{B}, \mathrm{P}<0.0001$; A vs. $\mathrm{C}, \mathrm{P}=0.001$; A vs. D, $\mathrm{P}=0.038$; A vs. $\mathrm{E}, \mathrm{P}=0.008)$. On the contrary, group $\mathrm{B}$ showed lower scores compared with all other groups (all $\mathrm{P}<0.0001$ ), indicating the most severe damage in spermatogenesis, because there was no pharmaceutical intervention. Finally, differences in Johnsen's score among groups C, D and E were not statistically significant (all $\mathrm{P}>0.99$ ), indicating spermatogenesis of equal quality. These groups treated with erythropoietin or sildenafil presented higher Johnsen's scores compared with 
A

B

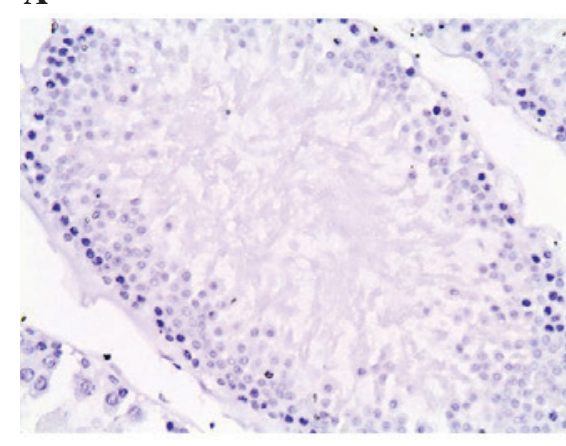

D

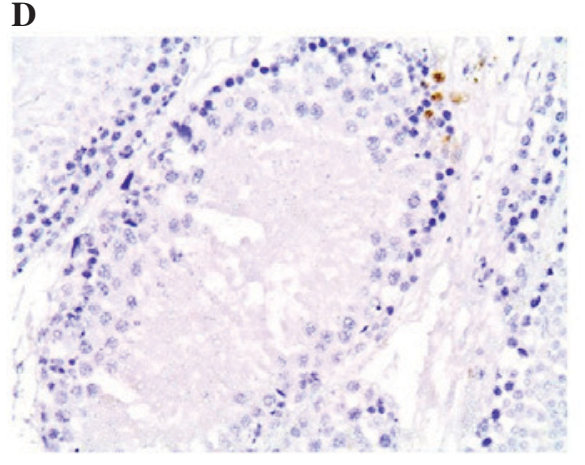

C

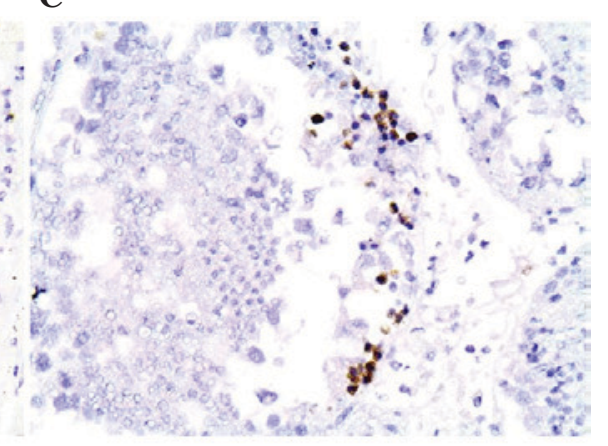

$\mathbf{E}$

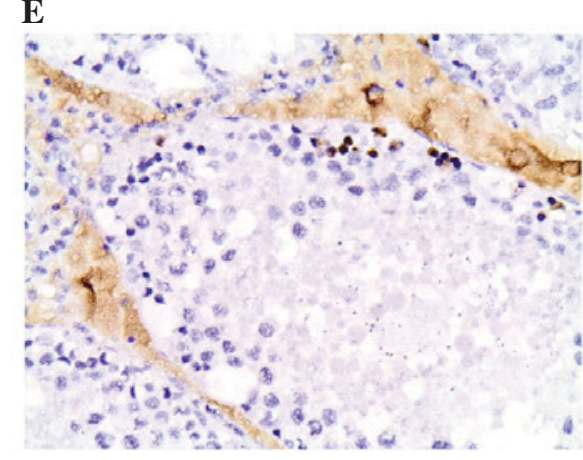

Figure 2. Histological images of immunohistochemical stain for cleaved caspase-3 in the (A) sham-operated, (B) torsion/detorsion without treatment, (C) torsion/detorsion + low-dose erythropoietin,(D) torsion/detorsion + high-dose erythropoietin, and (E) torsion/detorsion + sildenafil groups (original magnification, $\mathrm{x} 400)$.

group $\mathrm{B}$ (all $\mathrm{P}<0.0001)$, indicating better quality of spermatogenesis, but lower scores compared with group A (C vs. A, $\mathrm{P}=0.001 ; \mathrm{D}$ vs. $\mathrm{A}, \mathrm{P}=0.038$; $\mathrm{E}$ vs. $\mathrm{A}, \mathrm{P}=0.008)$, indicating lower quality of spermatogenesis.

Cleaved caspase-3 levels. Apoptosis in the rat testes was evaluated by estimating the cleaved caspase- 3 expression. Indicative histological images from each group are shown in Fig. 2. The control testes in group A presented the lowest levels of cleaved caspase-3 (mean percentage, $0.03 \%$; range, $0-0.06 \%$ ). By contrast, group B presented the highest levels of cleaved caspase-3 (mean percentage, 2.71\%; range, 2.24-3.22\%), and thus increased apoptosis. Furthermore, testes from groups C, D and E that were treated with erythropoietin or sildenafil exhibited moderate percentages of cleaved caspase-3 expression (group C: mean percentage, $1.124 \%$; range, $0.4-1.82 \%$; group D: mean percentage, $0.2934 \%$; range, $0.097-0.64 \%$; group E: mean percentage, $0.355 \%$; range, $0.06-0.81 \%$ ). Comparisons among the five groups revealed statistical significant differences regarding cleaved caspase-3 levels. In particular, group B presented higher cleaved caspase-3 levels compared with all the other groups (all $\mathrm{P}<0.0001$ ), indicating the most severe histological damage. Group C presented lower cleaved caspase-3 levels compared with group $\mathrm{B}(\mathrm{P}<0.0001)$, indicating lower degree of apoptosis. However, this group exhibited higher cleaved caspase-3 levels than groups $\mathrm{A}, \mathrm{D}$ and $\mathrm{E}(\mathrm{C}$ vs. $\mathrm{A}, \mathrm{P}=0.001$; $\mathrm{C}$ vs. $\mathrm{D}, \mathrm{P}=0.01$; $\mathrm{C}$ vs. $\mathrm{E}, \mathrm{P}=0.008$ ), indicating that the low-dose erythropoietin treatment did not reduce apoptosis following torsion/detorsion to the same extend as the high-dose erythropoietin or sildenafil treatments. By contrast, no significant differences were observed between the groups A, D and E concerning cleaved caspase-3 expression (all $\mathrm{P}>0.99$ ), indicating similar low levels of apoptosis in those treatment groups as in the sham-operated group.

Correlations among Cosentino's score, Johnsen's score and cleaved caspase-3 expression. A correlation analysis revealed that Cosentino's score, which depicts histological changes of the testis, was inversely correlated with Johnsen's score $(\mathrm{r}=-0.908 ; \mathrm{P}<0.0001)$, which depicts lesions in spermatogenesis. Furthermore, Cosentino's score was correlated with cleaved caspase-3 expression ( $r=0.909 ; \mathrm{P}<0.0001)$. Finally, Johnsen's score was inversely correlated with cleaved caspase-3 production $(\mathrm{r}=-0.815 ; \mathrm{P}<0.0001)$.

\section{Discussion}

Different pathogenic mechanisms are involved in acute testicular damage caused by torsion and detorsion, resulting in detrimental ischemia and reperfusion time-related injuries (4,5,7-9). Ischemia induces oxidative stress and germ cell death attributed to the reduction in oxygen supply, depletion of cellular energy and accumulation of toxic metabolites (29). The reperfusion injury is characterized by increased production of reactive oxygen and nitrogen species, and cause peroxidation, which may lead to disorganization of the cell structure and function (29). Different therapeutic agents have been investigated with the aim to reduce testicular ischemia/reperfusion injury. In particular, erythropoietin and sildenafil have been reported to reduce injury caused by ischemia/reperfusion in a variety of tissues and organs, including the spinal cord, kidney, liver, heart, lung, brain, intestine and retina in animals $(10,11)$.

Erythropoietin is a multiple functional endogenous mediator regarded as a general tissue-protective agent. The 
anti-inflammatory, anti-oxidative and anti-apoptotic properties of erythropoietin offer a protective effect against ischemia/reperfusion injury (10,30). Endothelial nitric oxide synthase, vascular endothelial growth factor, Bcl-2 and nuclear factor kappa B are some of the several pathways that are involved in the protective mechanisms (10). In addition, sildenafil has a protective role as well, particularly in myocardium. Sildenafil and other phosphodiesterase type-5 inhibitors cause vasodilation and release mediators of ischemic preconditioning, such as adenosine and/or bradykinin (11).

The role of erythropoietin and sildenafil against ischemia/reperfusion injury during testicular torsion and detorsion has been evaluated in rats in several studies $(16,21,31,32)$. The intraperitoneal injection of erythropoietin prior to detorsion decreases the histological damage of the testis by reducing the damage and the apoptotic rate of germinal cells, reducing the necrotic lesions of seminiferous tubules and preserving their morphology $(16,21,31,32)$. Erythropoietin also retains its protective role when it is administered soon after detorsion $(21,22)$. Concerning the underlying mechanisms, it is supported that erythropoietin-associated protection of testes exposed to oxidative stress may be attributed to the increased levels of glutathione and to the decreased levels of oxidative stress markers, including nitric oxide and malondialdehyde (31). The intraperitoneal injection of sildenafil prior to detorsion also reduces the apoptotic rates of germinal cells and prevents severe damage of seminiferous tubules $(17-20,33,34)$. Other phosphodiesterase type 5 inhibitors, such as vardenafil $(35,36)$ and udenafil $(34)$, also protect the testis from ischemia/reperfusion injury when administered prior to (valdenafil and udenafil) $(34,35)$ or soon after detorsion (valdenafil) (36). There are several studies indicating that sildenafil administration is able to induce anti-ischemic effect as evidenced by increased antioxidant enzymes levels, decreased nitric oxide and malondialdehyde levels, as well as germ cell apoptotic indices $(17,18,20,34)$. It has also been reported that the activity of several enzymes that protect against oxidative damage, including catalase, glutathione peroxidase and superoxide dismutase, is increased following sildenafil treatment $(17,18,20,34)$.

The present study confirmed the protective role of erythropoietin and sildenafil against the ischemia/reperfusion injury of the testis during torsion and detorsion in adult rats. Erythropoietin and sildenafil attenuated the deleterious effects of ischemia/reperfusion injury when they were injected intraperitoneally $30 \mathrm{~min}$ before detorsion. The two drugs reduced the histological damage, as it was assessed by Cosentino's score, with better-defined borders of seminiferous tubules and reduced number of apoptotic germinal cells, as compared with the untreated torsion/detorsion group. In addition, these treatments resulted in less affected spermatogenesis, as it was evaluated by Johnsen's score, when compared with the untreated group. In terms of the histological changes, the administration of high-dose erythropoietin $(3,000 \mathrm{IU} / \mathrm{kg})$ or sildenafil $(0.7 \mathrm{mg} / \mathrm{kg})$ provided equal results, whereas the administration of low-dose erythropoietin $(1,000 \mathrm{IU} / \mathrm{kg})$ yielded inferior results, although improvement was observed compared with the untreated torsion/detorsion group. However, neither drug provided histological images equal to those of the sham-operated control group. Regarding the quality of spermatogenesis, the administration of low-dose erythropoietin $(1,000 \mathrm{IU} / \mathrm{kg})$, high-dose erythropoietin $(3,000 \mathrm{IU} / \mathrm{kg})$ or sildenafil $(0.7 \mathrm{mg} / \mathrm{kg})$ yielded equal results, but without reaching the spermatogenesis quality observed in the sham-operated group. The findings of the present study are consistent with the results of other investigators reporting the loss of germ cells following ischemia/reperfusion injury and showing that pretreatment with erythropoietin and sildenafil prevented germ cell apoptosis in the rats $(4,10,11)$.

In the current study, apoptosis was confirmed by the increased levels of caspase-3 observed subsequent to detorsion in the untreated rats. Caspases are a family of proteases that propagate and coordinate programmed cell death. There are two caspase subcategories, including the initiator caspases that stimulate and initiate the apoptotic cascade, and the effector caspases that execute the cell death program in order to dismantle the cell. Caspase-3 is a major effector caspase (25-28), whose activity is increased in testicular ischemic injury (37). Neutrophils and macrophages recruited during reperfusion have been implicated as mediators of tissue injury, and due to their ability to release various toxic materials, such as oxygen-free radicals and inflammatory cytokines, they can induce testicular cell apoptosis (4,5,7-9). The current study results demonstrated that the levels of cleaved caspase-3, which is the activated form of the enzyme, are reduced when erythropoietin or sildenafil is administered in comparison with the untreated group. The administration of high-dose erythropoietin $(3,000 \mathrm{IU} / \mathrm{kg})$ or sildenafil $(0.7 \mathrm{mg} / \mathrm{kg})$ provided equal results, which were equivalent to those of the sham-operated group. On the other hand, the administration of low-dose erythropoietin $(1,000 \mathrm{IU} / \mathrm{kg})$ yielded inferior results, although improvement was observed compared with the untreated torsion/detorsion group. It is suggested that these findings correspond to the degree of activation of the apoptotic cascade, as the caspase-3 serves an important role in it (25-28). The reduced activation of caspase- 3 following erythropoietin administration has also been reported by other studies $(21,32)$.

In a previous study by our group, the beneficial effects of sildenafil $(0.7 \mathrm{mg} / \mathrm{kg})$ and low-dose erythropoietin $(1,000 \mathrm{mg} / \mathrm{kg})$ were demonstrated in rats subjected to testicular torsion and detorsion (15). Sildenafil provided better results compared with the low-dose erythropoietin $(1,000 \mathrm{mg} / \mathrm{kg})$; however, to the assessment of histological damages in the testis was only assessed by the Cosentino's score, providing a limitation to the study (15). Due to the addition of a high-dose erythropoietin treatment $(3,000 \mathrm{IU} / \mathrm{kg})$, as well as the assessment of spermatogenesis through the Johnsen's score and the apoptosis through the expression of cleaved caspase-3, the present study provides new findings that could not be identified in our previous study. Specifically, it was shown herein that, in terms of the histological changes and the prevention of apoptosis, the administration of high-dose erythropoietin $(3,000 \mathrm{IU} / \mathrm{kg})$ or sildenafil $(0.7 \mathrm{mg} / \mathrm{kg})$ provided equal results, whereas the administration of low-dose erythropoietin $(1,000 \mathrm{mg} / \mathrm{kg})$ yielded inferior results. In addition, regarding the quality of spermatogenesis, the administration of low-dose erythropoietin $(1,000 \mathrm{mg} / \mathrm{kg})$, high-dose erythropoietin $(3,000 \mathrm{IU} / \mathrm{kg})$ or sildenafil $(0.7 \mathrm{mg} / \mathrm{kg})$ yielded equal results.

In conclusion, erythropoietin and sildenafil protect the testis of adult rats against ischemia/reperfusion injury during 
torsion and detorsion. Strong evidence suggested that intraperitoneal injection of one of the two drugs prior to detorsion attenuated the acute testicular damage after ischemia and the deleterious effects of the reactive oxygen species produced by reperfusion. However, future research can be focused on dose-response investigations and studies with different methodological approaches in various organs and tissues under a clinical perspective.

\section{References}

1. Kapoor S: Testicular torsion: A race against time. Int J Clin Pract 62: 821-827, 2008

2. Gatti JM and Patrick Murphy J: Current management of the acute scrotum. Semin Pediatr Surg 16: 58-63, 2007.

3. Ringdahl E and Teague L: Testicular torsion. Am Fam Physician 74: 1739-1743, 2006.

4. Turner TT, Bang HJ and Lysiak JL: The molecular pathology of experimental testicular torsion suggests adjunct therapy to surgical repair. J Urol 172: 2574-2578, 2004.

5. Altavilla D, Romeo C, Squadrito F, Marini H, Morgia G, Antonuccio P and Minutoli L: Molecular pathways involved in the early and late damage induced by testis ischemia: Evidence for a rational pharmacological modulation. Curr Med Chem 19: 1219-1224, 2012.

6. Mellick LB: Torsion of the testicle: It is time to stop tossing the dice. Pediatr Emerg Care 28: 80-86, 2012.

7. Reyes JG, Farias JG, Henríquez-Olavarrieta S, Madrid E, Parraga M, Zepeda AB and Moreno RD: The hypoxic testicle: Physiology and pathophysiology. Oxid Med Cell Longev 2012: 929285, 2012.

8. Filho DW, Torres MA, Bordin AL, Crezcynski-Pasa TB and Boveris A: Spermatic torsion, reactive oxygen and nitrogen species and ischemia-reperfusion injury. Mol Aspects Med 25: 199-210, 2004

9. Lysiak JJ: The role of tumor necrosis factor-alpha and interleukin-1 in the mammalian testis and their involvement in testicular torsion and autoimmune orchitis. Reprod Biol Endocrinol 2: 9, 2004

10. Paschos N, Lykissas MG and Beris AE: The role of erythropoietin as an inhibitor of tissue ischemia. Int J Biol Sci 4 $161-168,2008$

11. Kukreja RC, Salloum F, Das A, Ockaili R, Yin C, Bremer YA FisherPW,Wittkamp M,Hawkins J,ChouE, et al: Pharmacological preconditioning with sildenafil: Basic mechanisms and clinical implications. Vascul Pharmacol 42: 219-232, 2005.

12. Fisher JW: Erythropoietin: Physiology and pharmacology update. Exp Biol Med (Maywood) 228: 1-14, 2003.

13. Li J, Fu P, Deleon M, French BA and French SW: The effect of Viagra (sildenafil citrate) on liver injury caused by chronic ethanol intragastric feeding in rats. Exp Mol Pathol 78: 101-108, 2005.

14. Zhang RL, Zhang Z, Zhang L, Wang Y, Zhang C and Chopp M: Delayed treatment with sildenafil enhances neurogenesis and improves functional recovery in aged rats after focal cerebral ischemia. J Neurosci Res 83: 1213-1219, 2006.

15. Zavras N, Kostakis ID, Sakellariou S, Damaskos C, Roupakas E, Tsagkari E, Spartalis E, Velaoras K, Dontas IA and Karatzas T: Comparison of erythropoietin and sildenafil protective role against ischemia/reperfusion injury of the testis in adult rats. Int Urol Nephrol 46: 731-736, 2014.

16. Köseoğlu B, Yilmaz E, Ceylan K, Uzun E, Bayram I and Hizli F: The protective effect of erythropoietin infusion on testicular torsion/detorsion: An experimental study. Int Urol Nephrol 41: 85-91, 2009.
17. Beheshtian A, Salmasi AH, Payabvash S, Kiumehr S, Ghazinezami B, Rahimpour S, Tavangar SM and Dehpour AR: Protective effects of sildenafil administration on testicular torsion/detorsion damage in rats. World J Urol 26: 197-202, 2008.

18. Yildiz H, Durmus AS, Şimşek H and Yaman M: Dose-dependent protective effect of sildenafil citrate on testicular injury after torsion/detorsion in rats. Andrologia 44 (Suppl 1): S300-S306, 2012

19. Yildiz H, Durmus AS, Simsek H and Yaman I: Effects of sildenafil citrate on torsion/detorsion-induced changes in red blood cell and plasma lipid peroxidation, antioxidants, and blood hematology of male rats. Eur J Obstet Gynecol Reprod Biol 159: 359-363, 2011.

20. Yildiz H, Durmus AS, Simşek H and Yaman M: Protective effect of sildenafil citrate on contralateral testis injury after unilateral testicular torsion/detorsion. Clinics (Sao Paulo) 66: 137-142, 2011.

21. Yazihan N, Ataoglu H, Koku N, Erdemli E and Sargin AK: Protective role of erythropoietin during testicular torsion of the rats. World J Urol 25: 531-536, 2007.

22. Rashed FK, Ghasemi B, Deldade Mogaddam H and Mesgari M: The effect of erythropoietin on ischemia/reperfusion injury after testicular torsion/detorsion: A randomized experimental study. ISRN Urol 2013: 351309, 2013.

23. Cosentino MJ, Nishida M, Rabinowitz R and Cockett AT: Histological changes occurring in the contralateral testes of prepubertal rats subjected to various durations of unilateral spermatic cord torsion. J Urol 133: 906-911, 1985.

24. Johnsen SG: Testicular biopsy score count-a method for registration of spermatogenesis in human testes: Normal values and results in 355 hypogonadal males. Hormones 1: 2-25, 1970.

25. Parrish AB, Freel CD and Kornbluth S: Cellular mechanisms controlling caspase activation and function. Cold Spring Harb Perspect Biol 5: pii: a008672, 2013.

26. Mcllwain DR, Berger T and Mak TW: Caspase functions in cell death and disease. Cold Spring Harb Perspect Biol 5: a008656, 2013.

27. Alenzi FQ, Lotfy M and Wyse R: Swords of cell death: Caspase activation and regulation. Asian Pac J Cancer Prev 11: 271-280, 2010.

28. Pop C and Salvesen GS: Human caspases: Activation, specificity, and regulation. J Biol Chem 284: 21777-21781, 2009.

29. Carden DL and Granger DN: Pathophysiology of ischaemia-reperfusion injury. J Pathol 190: 255-266, 2000.

30. Joyeux-Faure M: Cellular protection by erythropoietin: new therapeutic implications? J Pharmacol Exp Ther 323: 759-762, 2007.

31. Akcora B, Altug ME, Kontas T and Atik E: The protective effect of darbepoetin alfa on experimental testicular torsion and detorsion injury. Int J Urol 14: 846-850, 2007.

32. Ergur BU, Kiray M, Pekcetin C, Bagriyanik HA and Erbil G: Protective effect of erythropoietin pretreatment in testicular ischemia-reperfusion injury in rats. J Pediatr Surg 43: 722-728, 2008.

33. Halawa AM: Effect of sildenafil administration on ischemia/reperfusion of the testis in adult Albino rat light and electron microscopic study. Egypt J Histol 33: 380-395, 2010

34. Özgür BC, Telli O, Yuceturk CN, Sarici H, Ozer E, Surer H, Kilinc AS, Hucumenoglu S and Eroglu M: The effect of sildenafil and udenafil on testicular damage following ischemia-reperfusion injury in rats. J Urol 192: 1272-1277, 2014.

35. Istanbulluoglu MO, Zor M, Celik A, Cicek T, Basal S, Ozgok A, Ustun $\mathrm{H}$ and Ozgok $\mathrm{Y}$ : Effects of vardenafil on testicular torsion/detorsion damage: An experimental study in pigs. Urol Int 86: 228-232, 2011.

36. Erol B, Tokgoz H, Hanci V, Bektas S, Akduman B, Yencilek F, Mungan $\mathrm{G}$ and Mungan A: Vardenafil reduces testicular damage following ischemia/reperfusion injury in rats. Kaohsiung J Med Sci 25: 374-380, 2009.

37. Lysiak JJ, Turner SD and Turner TT: Molecular pathway of germ cell apoptosis following ischemia/reperfusion of the rat testis. Biol Reprod 63: 1465-1472, 2000. 